\title{
Reconstruction of Fresnel holograms using partial wave front information
}

\author{
R. Tudela, E. Martín-Badosa, I. Labastida, S. Vallmitjana and A. Carnicer \\ Departament de Física Aplicada i Òptica. Universitat de Barcelona. \\ Avda. Diagonal 647, E08028 Barcelona, Spain
}

\begin{abstract}
In this work we analyze the behavior of complex information in Fresnel domain taking into account the limited capability to display complex transmittance values of current liquid crystal devices, when used as holographic displays. In order to do this analysis we compute the reconstruction of Fresnel holograms at several distances using the different parts of the complex distribution (real and imaginary parts, modulus and phase) as well as using the full complex information adjusted with a method that combines two real configurations of the devices in an adding architecture. The RMS error between the amplitude of these reconstructions and the original amplitude is used to evaluate the quality of the information displayed. The results show different behavior for the reconstructions using the different parts of the complex distribution and using the combined method of two devices. There is possible to obtain better reconstructions using two devices, which added configurations densely cover the complex plane. Simulated and experimental results are also presented.
\end{abstract}

Keywords: Fresnel diffraction, wave front propagation, holography, liquid crystal devices, optical image processing

\section{INTRODUCTION}

In many optical applications it is necessary to use complex information, which have to be accurately controlled and displayed on real time. The use of liquid crystal devices (LCD) as spatial light modulators (SLM) has became a generalized way to obtain real time manipulation of wave fronts, which implies the use of complex functions in order to modify the amplitude and phase of a wave. Most of the present LCDs provide a wide range of operating modes but none of them allows the complete display of a full complex plane. It makes necessary to develop some strategies to achieve full complex modulation. A first approach is to use only one display where the complex information has to be codified, using classical holographic methods ${ }^{1}$ or new approaches. ${ }^{2-4}$ These methods entail a loss of resolution when displaying the information on the devices. A second strategy is to couple two SLMs to obtain the full complex modulation, taking into account the coupling optical setup and the modulation capabilities of each device. Juday et $a l^{5}$ and Gregory et al proposed the two common architectures to combine two LCDs, one combining amplitude and phase as a product, which can be achieved with different combinations and devices ${ }^{7-9}$ and another by combining the real and imaginary parts as an addition. ${ }^{10}$

In this work we use the additive architecture to reconstruct Fresnel holograms, coupling two devices with other configurations than real-only and imaginary-only. First we present a way to represent a complex number as the sum of two phases. This method can not be used directly with devices not able to modulate phase ideally and have to be adapted to the addition of real configurations of LCDs. We present simulated results of this method to adjust complex functions with the combination of different real configurations in an adding architecture and we study de information on Fresnel holograms when using only a part of the complex information, or using two devices coupled with configurations other than real-only and phase-only to reconstruct the object. Finally we present some experimental results and the conclusions.

Correspondence to Raúl Tudela: e-mail: raul@fao.ub.es, telephone: +34934021203 


\section{COMPLEX NUMBERS AS PHASE COMBINATION}

Complex numbers could be represented in different ways, usually they are presented as the addition of the real and imaginary parts or as the product of the modulus and the phase:

$$
z=a+i b=M e^{i \theta} .
$$

Another way to represent a complex number is as the addition of two phases. This is possible if the modulus of the complex number is below to two (addition of two numbers with modulus below to one), then we have,

$$
z=e^{i \phi_{1}}+e^{i \phi_{2}}
$$

To demonstrate this possibility of combination we show the relationship between the phases $\phi_{1}$ y $\phi_{2}$ in equation (2) and the real and imaginary parts in equation (1). In order to do that we develop equation (2 into an addition of sinus and cosinus,

$$
z=\left(\cos \phi_{1}+\cos \phi_{2}\right)+i\left(\sin \phi_{1}+\sin \phi_{2}\right)
$$

separating the real and imaginary parts, we have,

$$
\begin{gathered}
a=\cos \phi_{1}+\cos \phi_{2}=2 \cos \left(\frac{\phi_{1}+\phi_{2}}{2}\right) \cos \left(\frac{\phi_{1}+\phi_{2}}{2}\right), \\
b=\sin \phi_{1}+\sin \phi_{2}=2 \sin \left(\frac{\phi_{1}+\phi_{2}}{2}\right) \cos \left(\frac{\phi_{1}+\phi_{2}}{2}\right),
\end{gathered}
$$

and by dividing the imaginary part and real part we obtain,

$$
\frac{b}{a}=\tan \left(\frac{\phi_{1}+\phi_{2}}{2}\right)
$$

on the other hand, adding the square of the real and imaginary parts we have,

$$
\begin{gathered}
a^{2}=\cos ^{2} \phi_{1}+\cos ^{2} \phi_{2}+2 \cos \phi_{1} \cos \phi_{2}, \quad b^{2}=\sin ^{2} \phi_{1}+\sin ^{2} \phi_{2}+2 \sin \phi_{1} \sin \phi_{2}, \\
a^{2}+b^{2}=2+2\left(\cos \phi_{1} \cos \phi_{2}+\sin \phi_{1} \sin \phi_{2}\right)=2+2 \cos \left(\phi_{1}-\phi_{2}\right)=4 \cos ^{2}\left(\frac{\phi_{1}-\phi_{2}}{2}\right) .
\end{gathered}
$$

Finally, combining equations (5) y (7) we obtain the relationship to obtain the phases that added will give us the complex number using its real and imaginary parts,

$$
\phi_{1}=\arctan \frac{b}{a}+\arccos \sqrt{\frac{a^{2}+b^{2}}{4}}, \quad \phi_{2}=\arctan \frac{b}{a}-\arccos \sqrt{\frac{a^{2}+b^{2}}{4}},
$$

that also can be expressed in terms of modulus and phase,

$$
\phi_{1}=\theta+\arccos \frac{M}{2}, \quad \phi_{2}=\theta-\arccos \frac{M}{2} .
$$




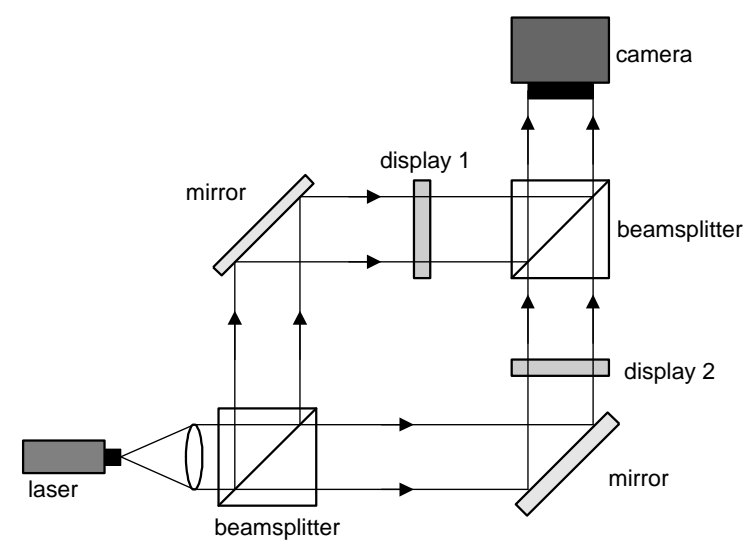

(a)

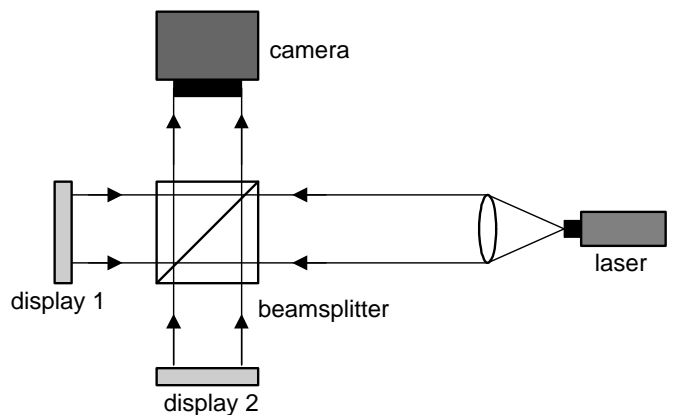

(b)

Figure 1. Architectures to obtain the addition of two LCDs (a) transmissive devices and (b) reflexive devices

\section{CONFIGURATIONS ADDITION OF LIQUID CRYSTALS}

We can express a complex function as the sum of two functions of only phase. This allows to represent complex information through the addition of two only phase parts, which can be displayed on liquid crystal devices (LCDs) used as spatial light modulators. Depending on the characteristics of the devices we can obtain the addition using different architectures, as shown in figure 1.

We can use this system to display Fresnel holograms, computed by backpropagating an object $f(x, y)$ to a distance $-d$, using equation 10 , and then separating the obtained distribution, $U(x, y,-d)$, into the sum of two phases. The problem appears when the two obtained phases must be displayed onto real liquid crystal devices, which involves the adjustment of the phase values to the ones available in the modulators. If the device do not have a ideal only phase configuration, which can display all the possible phase values with amplitude one, the values that will be shown do not correspond to the phase values that added give the complex information to be displayed. Then the reconstruction obtained with devices without an ideal only phase configuration will be very noisy and inaccurate.

$$
\begin{array}{r}
U(x, y,-d)=\mathcal{F} \text { resnel }[f(x, y),-d]= \\
=\frac{e^{-\frac{i 2 \pi d}{\lambda}}}{i \lambda d} \int_{-\infty}^{\infty} f\left(x^{\prime}, y^{\prime}\right) \exp \left[-\frac{i \pi}{\lambda d}\left(\left(x-x^{\prime}\right)^{2}+\left(y-y^{\prime}\right)^{2}\right)\right] d x^{\prime} d y^{\prime}
\end{array}
$$

An alternative to adjust a complex function to the liquid crystal devices considering their modulations capabilities is to adjust the complex values directly to a combination of the configurations of the two devices, without doing the separation into phases. Doing this we can adapt the idea for obtaining a complex number as the sum of other two numbers using the addition of two displays (equation (11)), but in this case the complex numbers to be added must belong to the modulation curves $(M C)$ of the devices in the available configurations. Thus each modulator can work in a suitable configuration and adding the curves of the two devices (point to point) we obtain a new curve that covers more zone in the complex plane that using the two separates curves.

$$
z \simeq z_{1}+z_{2} \quad \text { with } \quad z_{1} \in M C_{1} \text { and } \quad z_{2} \in M C_{2}
$$




\section{SIMULATIONS WITH REAL CONFIGURATIONS}

We have applied this technique using real configurations of liquid crystal devices, we use three configurations obtained for devices of a EPSON VGA video projector EMP-3000, the first one (figure 2(a)) is a phase mostly configuration (pmvga) with a phase modulation of $1.60 \pi$ and a contrast ratio of $5.4: 1$, the second configuration is a $0-\pi$ half phase curve (pelvga) with a phase modulation of $0.98 \pi$ and a contrast ratio of $1.1: 1$ (figure $2(\mathrm{~b})$ ), and the last curve is a high contrast configuration (hcvga) with a phase modulation of $0.75 \pi$ and a contrast ratio of $124: 1$ (figure 2(c)). Combining these three curves, by adding each point of one configuration with all the points of another, we can obtain the different added curves shown in figure 3. In these curves are represented the values that are accessible to be modulated with the use of two devices in an adding configuration. The complex function to be represented has to be adjusted to these dense distributions, the method used is a minimum Euclidean distance criterion (MED), the amplitude values of the function are normalized to de maximum amplitude of the curve, and then each point is adjusted to his nearest point in the curve, that can be achieve by the addition of two points corresponding to the real configurations of the devices. The combination that covers more densely the normalized complex plane will give the better results to represent a complex function.

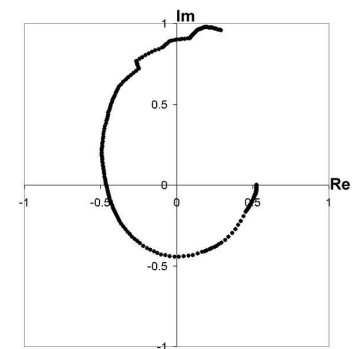

(a)

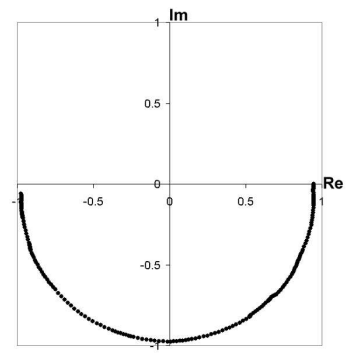

(b)

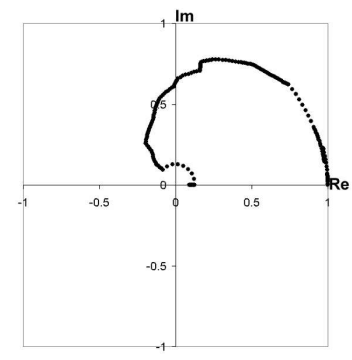

(c)

Figure 2. Configuration curves of the VGA devices: (a) phase-mostly (pmvga), (b) half-phase (pelvga), and (c) high contrast (hcvga)

To study he different combinations we have used a distribution of the unity complex plane, shown in figure 4, this function is adjusted to the different combinations obtained from the three modulation curves of our devices, and with these adjusted distributions we obtain the images of the amplitude, phase, real and imaginary parts that are used to calculate the RMS (root mean squared) error (see equation 12) between each image and its respective ideal one. These results are shown in the graphic of figure 5 , where we can see the difference between each combination, for the real, imaginary, amplitude and phase parts.

$$
R M S=\sqrt{\frac{1}{N} \sum_{i=1}^{N}\left(A_{\text {adjusted }}[i]-A_{\text {ideal }}[i]\right)^{2}} \quad \text { with } \quad N=\text { number of pixels }
$$

The lower errors are obtained for the pelvga+hcvga and pmvga+pelvga combinations, which have values represented more uniformly over the complex plane, whilst the biggest errors are obtained using the pelvga+pelvga and hcvga+hcvga combinations that corresponds to configurations where the accessible points are not distributed uniformly in the plane. In this work we have chosen the pmvga+hcvga combination, as it presents an intermediate behavior, that is achieve by combining two different configurations of LCDs, one of phase mostly and one of high contrast. In figure 6 there are shown the images of the unity complex plane adjusted with this adding combination.

We have used the combination pmvga+hcvga to reconstruct Fresnel holograms, as a complex function to be represented using and adding architecture. The object to be reconstructed is the logo shown in figure 7(a), that represents the shadow of a building and two letters. With this image is calculated a Fresnel hologram using 


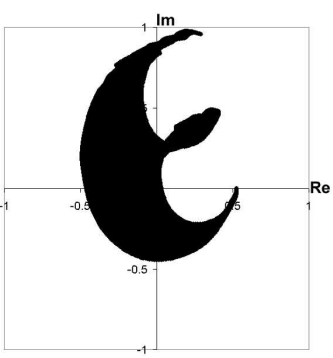

(a)

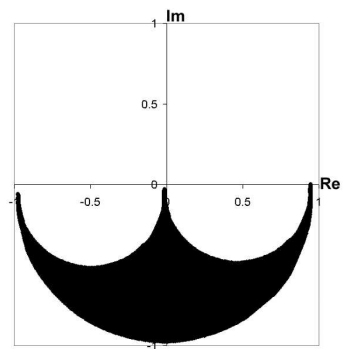

(d)

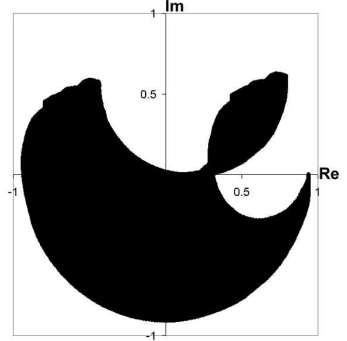

(b)

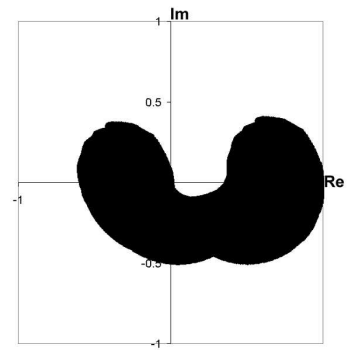

(e)

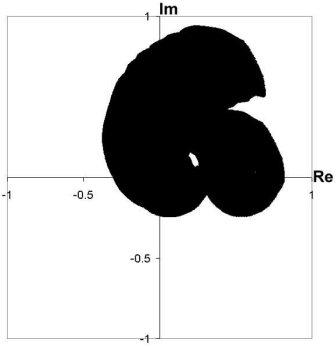

(c)

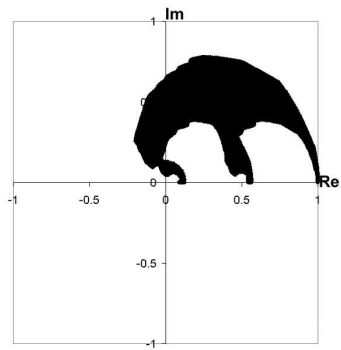

(f)

Figure 3. Accessible values with de addition of two configuration curves: (a) pmvga+pmvga, (b) pmvga+pelvga, (c) pmvga+hcvga, (d) pelvga+pelvga, (e) pelvga+hcvga, and (f) hcvga+hcvga

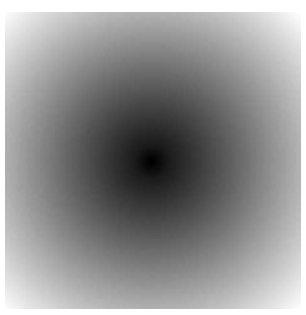

(a)

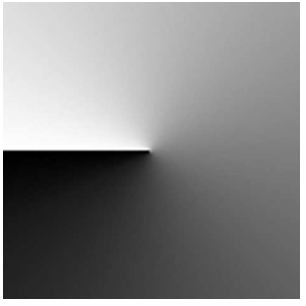

(b)

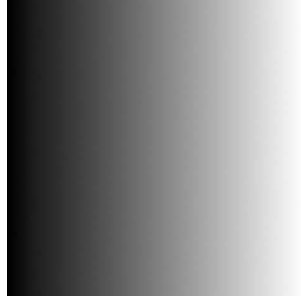

(c)

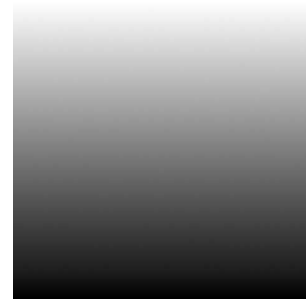

(d)

Figure 4. Images of the ideal unity complex plane distribution: (a) amplitude, (b) phase, (c) real part, and (d) imaginary part

equation 10 to reconstruct the object at $849 \mathrm{~mm}$. Adjusting this hologram using the combining method with the pmvga+hcvga configuration we obtain the reconstruction shown in figure $7(\mathrm{~b})$, that shows a good agreement with the original object. If we use only one of the single configurations we obtain the reconstructions shown in figures $7(\mathrm{c})$, using the phase mostly configuration (pmvga), and 7(d), using the high contrast one (hcvga), which have less quality than the obtained using the combined configuration.

Finally, we calculate the RMS error of the reconstructed amplitude of the logo of figure 7(a) at several distances, in figure 8(a) there are the results using only part of the complex information without adjusting to any configuration. We can see as the reconstructions using only the phase has a constant error for all the distances whilst the amplitude reconstruction error increases with the distance going over the phase reconstruction error. This is because the original image used is a binary image, in case of using a grey image with more details this result varies and the amplitude reconstruction error is lower than the phase one. The errors of the real and imaginary parts oscillates between the two curves shown in the graphic 8(a). In figure 8(b) there is a detail of this oscillations in a small zone around $849 \mathrm{~mm}$, the period of this oscillation is $\lambda / 2$ caused by the constructive of destructive interferences for the real or imaginary parts depending of the distance, for a detailed explanation 


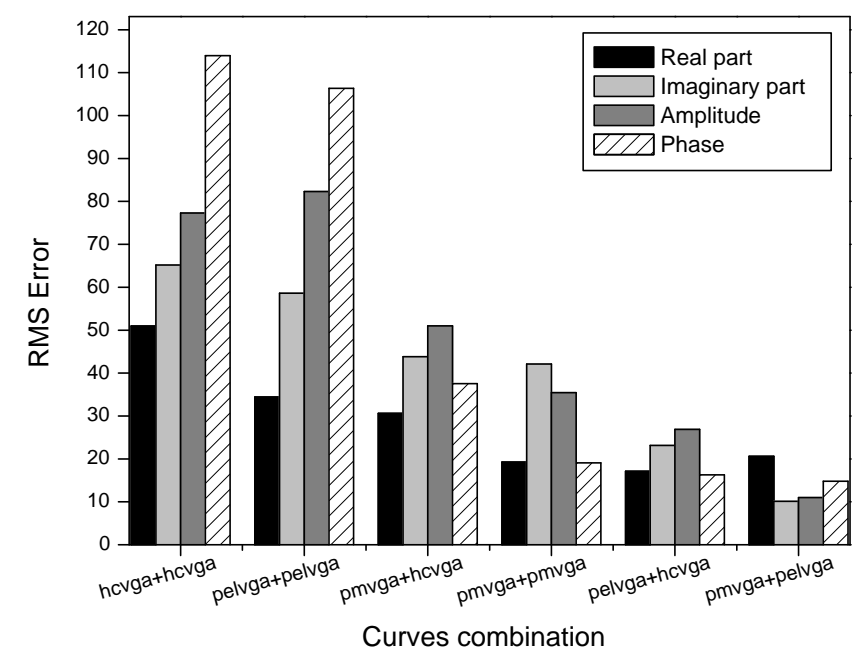

Figure 5. RMS errors between ideal complex plane images and the adjusted with the different combinations of the curves

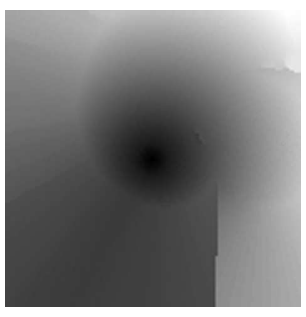

(a)

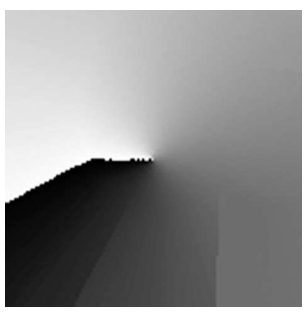

(b)

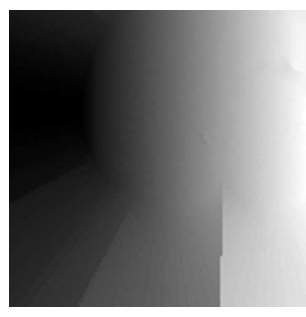

(c)

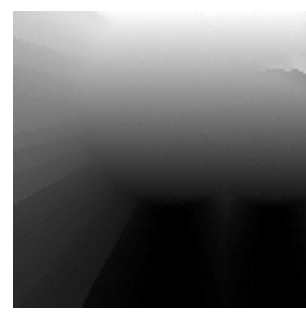

(d)

Figure 6. Images of the unity complex plane distribution adjusted to the combination pmvga+hcvga: (a) amplitude, (b) phase, (c) real part, and (d) imaginary part

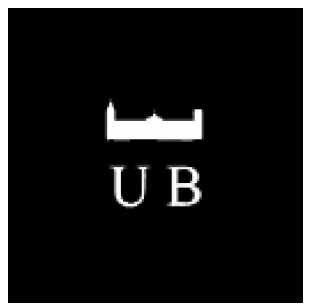

(a)

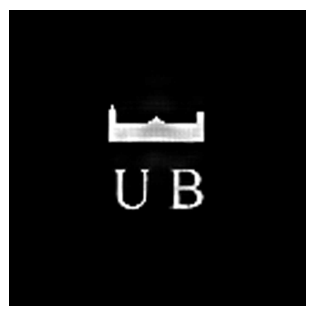

(b)

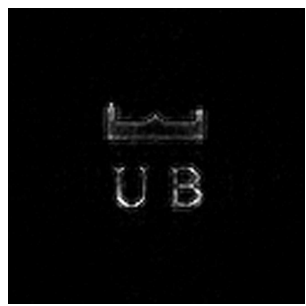

(c)

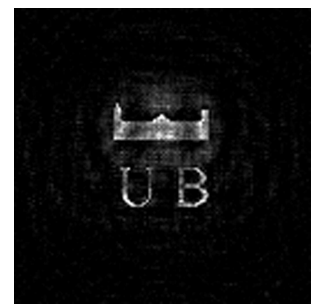

(d)

Figure 7. Images of (a) the original object to be reconstructed (logo with building and letters), (b) simulated reconstruction at $849 \mathrm{~mm}$ using the adding method with the pmvga and hcvga curves, (c) simulated reconstruction using only the pmvga curve and (d) simulated reconstruction using only the hcvga curve

see for instance Tudela et al..$^{10}$ The difference between the lower and top errors of the oscillations depends on the image, in the case of a binary image this distance is lower than in the case of a more detailed image with 
different grey levels.

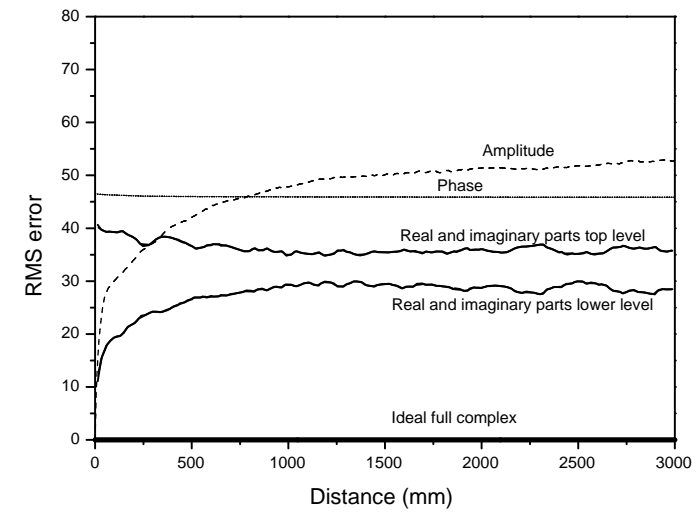

(a)

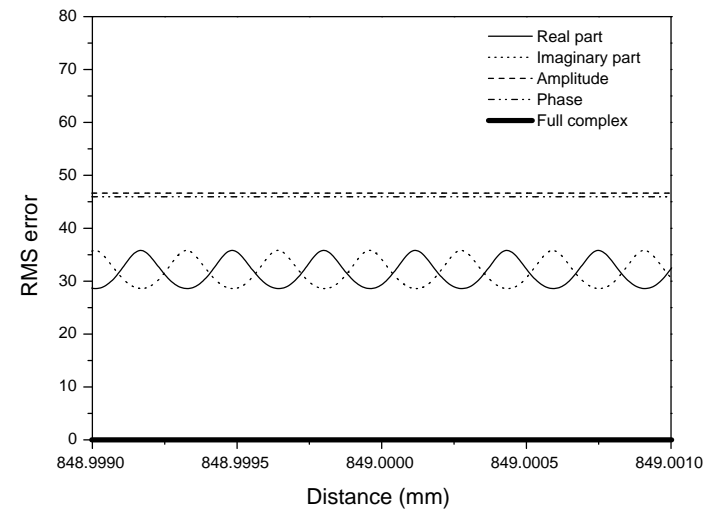

(b)

Figure 8. (a) RMS errors from 0 to $3000 \mathrm{~mm}$ for the reconstructed amplitude of the logo image (see figure 7 (a)) using the different parts without adjust and (b) detail of the oscillations of the RMS errors around $849 \mathrm{~mm}$

In figure 9 (a) there are plotted the RMS errors of the amplitude reconstructed using the adding method with the pmvga+hcvga configuration. The error oscillates between the two curves represented, but always is lower than the case of using only one part of the complex function without adjusting. The oscillations are shown in detail in figure 9(b) where the amplitude error is plotted in a small zone around $849 \mathrm{~mm}$. In this case the fundamental period of the oscillations is $\lambda$. Using other combinations of configuration to adjust the complex function using the adding method we obtain oscillations that depends on the curves used, but the fundamental periods obtained with a Fourier analysis are always integer fractions of $\lambda$. In all cases the lower error obtained using a combined method is better than the lower error using part of the complex information in the ideal case. In the graphics of figure 9 there are also plotted for completeness the errors of the amplitude reconstructions of the object using only the real part adjusted with the pmvga configuration and using only the imaginary part adjusted with the hcvga curve. In these cases we can see that the error when adjusting the data to real configurations of the devices and using only one device is bigger than in the ideal case, and how the oscillations of these adjustments determine the oscillations in the combined case.

\section{EXPERIMENTAL RESULTS}

We have also designed an optical set-up using two VGA devices removed from an EPSON EMP-3000 videoprojector. It consists of the two modulators arranged in an interferometric configuration as shown in figure 1(a). We use a He-Ne laser source of $632.8 \mathrm{~nm}$ and a CCD camera that can be displaced to the position where the object is reconstructed, at a distance from both SLMs equal to the backpropagated distance of the computed Fresnel hologram. We have reconstructed the object shown in figure 7(a) at the same distance that was performed in the simulation. In figure 10 there is the experimental reconstruction using the full complex information adjusted with the adding method to the pmvga+hcrga configuration. The loss of quality is due to experimental difficulties such as non-uniform illumination, noise introduced by the polarizing elements used to obtain the different configurations of the modulators, and the possible lack of parallelism between the panels and misalignments.

\section{CONCLUSIONS}

In this work we have analyzed the behavior of complex information in Fresnel domain taking into account the limited capability to display complex transmittance values of current liquid crystal devices, when used as 


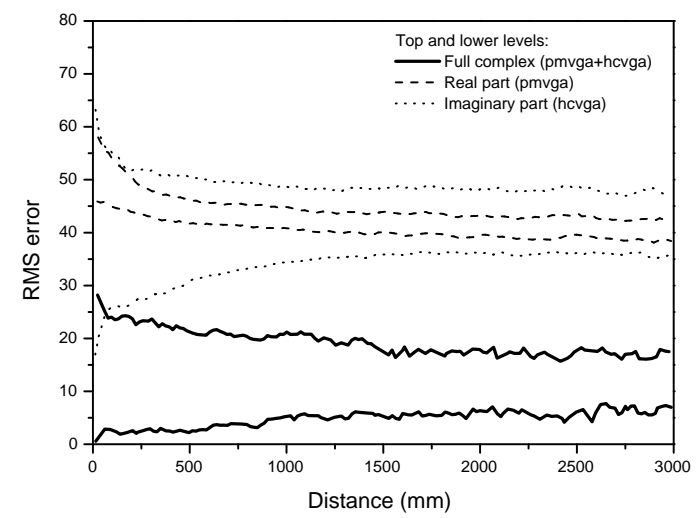

(a)

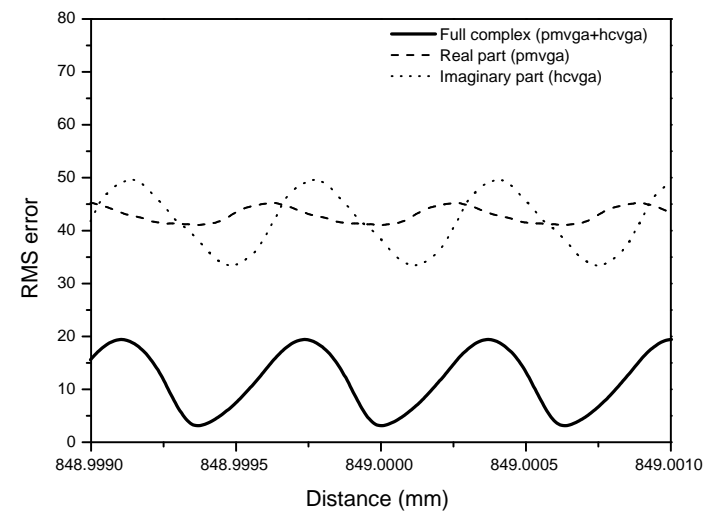

(b)

Figure 9. (a) RMS errors from 0 to $3000 \mathrm{~mm}$ for the reconstructed amplitude of the logo image (see figure 7(a)) using the adding method with the pmvga and hcvga curves or just the real or imaginary part adjusted to each curve respectively and (b) detail of the oscillations of the RMS errors around $849 \mathrm{~mm}$

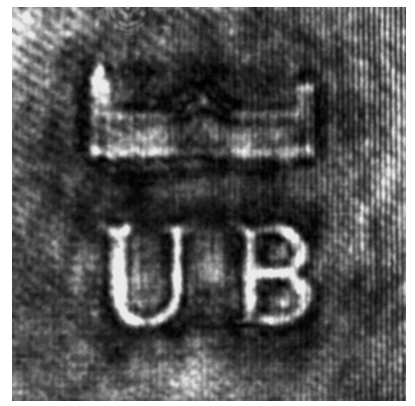

Figure 10. Experimental reconstruction of the image of the logo (figure $7(\mathrm{a})$ ) at $849 \mathrm{~mm}$ using the adding method with the pmvga+hcvga curve

holographic displays. We have introduced a way to represent complex functions as sum of two phases. This method can not be used directly with liquid crystal devices not able to modulate phase ideally. In order to adapt this method to real configurations we have devised an alternative to adjust a complex function to a combination of the configurations of two modulators in an adding architecture. To analyze this procedure we have used three real configurations of VGA panels and present the RMS error of the adjustment of the ideal unity complex plane to the six possible combinations obtained with these configurations, the best results are obtained when the combined curve covers more densely and uniformly the normalized complex plane.

We have computed the reconstruction of Fresnel holograms at several distances using the different parts of the complex distribution (real and imaginary parts, amplitude and phase) as well as using the full complex information with the combined configuration obtained with the phase mostly and the high contrast curves. The RMS error between the amplitude of these reconstructions and the original amplitude is calculated to evaluate the quality of the information displayed. The results show different behavior for the reconstructions using the different parts of the complex distribution without adjusting to any configuration: when using only the phase we obtain a constant but poor reconstruction for all the distances, whilst with the amplitude the quality of the reconstructions is higher for short distances and decreases with the distance, giving worst reconstructions than with the phase in the case of binary images. On the other hand, the real and the imaginary part have a 
complementary behavior: their reconstruction quality oscillates, with a period of half the wavelength, between a top and a lower level. When the real part gives a maximum of the RMS error the imaginary part gives a minimum and vice versa. In the case of using the real configurations of the modulators with the combined method the RMS error also oscillates but gives better reconstructions than using only part of the complex function. The experimental results using this method in an optical set up present good agreement with the simulated ones, and show that is possible to obtain full complex modulation by combining two configurations of the modulators that added will cover densely great part of the complex plane.

\section{ACKNOWLEDGMENTS}

This paper has been partially funded by the CICYT (Comisión Interministerial de Ciencia Y Tecnología) through project DPI 2001-3365 and by the CIRIT (Comissió Interdepartamental de Recerca I Tecnologia) through project 2001SGR00079.

\section{REFERENCES}

1. G. Tricoles, "Computer generated holograms: an historical review," Applied Optics 26, pp. 4351-4360, 1987.

2. S. Serati and K. Bauchert, "Sampling technique for achieving full unit-circle coverage using a real-axis spatial light modulator," in Optical Pattern Recognition X, D. P. Casasent, ed., Proc. SPIE 3715, pp. 112-119, 1999.

3. P. Birch, R. Young, C. Chatwin, M. Farsari, D. Budgett, and J. Richardson, "Fully complex optical modulation with an analogue ferroelectric liquid crystal spatial light modulator," Optics Communications 175, pp. 347-352, 2000.

4. J. A. Davis, K. O. Valadéz, and D. M. Cottrell, "Encoding amplitude and phase information onto a binary phase-only spatial light modulator," Applied Optics 42, pp. 2003-2008, 2003.

5. R. D. Juday and J. M. Florence, "Full complex modulation with two one-parameter slm's," in Wave Propagation and Scattering in Varied Media II, V. K. Varadan, ed., Proc. SPIE 1558, pp. 499-504, 1991.

6. D. A. Gregory, J. C. Kirsch, and E. C. Tam, "Full complex modulation using liquid-crystal televisions," Applied Optics 31, pp. 163-164, 1992.

7. J. Amako, H. Miura, and T. Sonehara, "Wave-front control using liquid-crystal devices," Applied Optics 32, pp. 4323-4329, 1993.

8. L. G. Neto, D. Roberge, and Y. Sheng, "Full-range, continous, complex-modulation by the use of two coupled-mode liquid-crystal televisions," Applied Optics 35, pp. 4567-4576, 1996.

9. R. Tudela, I. Labastida, E. Martín-Badosa, S. Vallmitjana, I. Juvells, and A. Carnicer, "A simple method for displaying Fresnel holograms on liquid crystal panels," Optics Communications 214, pp. 107-114, 2002.

10. R. Tudela, E. Martín-Badosa, I. Labastida, S. Vallmitjana, I. Juvells, and A. Carnicer, "Full complex Fresnel holograms displayed on liquid crystal devices," Journal of Optics A: Pure and Applied Optics -, pp. -, 2003. 